\title{
Spontaneous regression of a mediastinal thymoma
}

Tsai-Wang Huang, MD, ${ }^{\mathrm{a}}$ Yung-Lung Cheng, PhD, ${ }^{\mathrm{a}}$ Jen-Chih Chen, MD, ${ }^{\mathrm{a}}$ Wen-Chiuan Tsai, MD,${ }^{\mathrm{b}}$ Hung Chang, PhD, and Shih-Chun Lee, MD, ${ }^{\text {a }}$ Taipei, Taiwan

Thymoma is a common tumor in the anterior mediastinum. Thymoma may be symptomatic, but $20 \%$ to $30 \%$ of affected patients have symptoms related to compression of the mediastinum. Surgical excision is the keystone of therapy. Reports of the spontaneous regression of thymoma are rare in the English-language literature. Here we present a case of spontaneous remission of a mediastinal thymoma and a review of the literature.

\section{CLINICAL SUMMARY}

A 52-year-old woman had been admitted to our department 4 years previously because of an incidental abnormal finding on a chest radiograph during a physical checkup. The patient reported no history of disease. Computed tomography $(\mathrm{CT})$ of the chest revealed a well-defined mass located in the prevascular space (Figure 1, $A$ ). There were no symptoms of myasthenia gravis, such as weakness, ptosis, or diplopia. Her laboratory test results showed no significant abnormality, including her carcinoembryonic antigen, $\alpha$-fetoprotein, and $\beta$-human chorionic gonadotropin levels and

\footnotetext{
From the Division of Thoracic Surgery ${ }^{\mathrm{a}}$ and the Department of Pathology, ${ }^{\mathrm{b}}$ TriService General Hospital, National Defense Medical Center, Taipei, Taiwan.

Received for publication March 17, 2008; revisions received April 2, 2008; accepted for publication April 12, 2008.

Address for reprints: Shih-Chun Lee, MD, Division of Thoracic Surgery, Tri-Service General Hospital, 325, Section 2, Cheng Gong Rd, Taipei 114, Taiwan (E-mail: leesc001@yahoo.com.tw).

J Thorac Cardiovasc Surg 2009;137:1277-8 $0022-5223 / \$ 36.00$

Copyright (c) 2009 by The American Association for Thoracic Surgery doi:10.1016/j.jtcvs.2008.04.012
}

her anti-acetylcholine receptor antibody titer. Electrophysiologic examinations yielded normal results. The patient declined any further evaluation except a CT-guided biopsy. The histologic analysis was consistent with a World Health Organization classification type B2 thymoma (Figure 2). The patient elected not to receive any treatment and was followed up in the outpatient department every 3 months with no treatment. Three years later, CT of the chest did not reveal any mediastinal tumor (Figure $1, B$ ), and there was no radiologically apparent recurrence during a follow-up visit after 1 year.

\section{DISCUSSION}

Thymoma is a common anterior mediastinal tumor that is derived from thymic epithelial cells. The treatment of thymoma depends on its clinical presentation. CT scanning provides important assessment of the extent of the primary tumor, and biopsy should be performed when the tumor is large and not encapsulated. Surgical excision is the keystone of therapy. Spontaneous regression of thymomas is rare, with only two cases reported in the English-language literature. ${ }^{1,2} \mathrm{Kuo}^{1}$ described sclerosing thymoma as a possible phenomenon in patients with myasthenia gravis in remission, with arteriosclerosis and apoptosis being possible mechanisms. Okagawa and colleagues ${ }^{2}$ described the phenomenon of thymoma with spontaneous remission seen in imaging, with necrosis of the tumor probably caused by its rapid enlargement from an unknown cause. Okagawa and colleagues ${ }^{2}$ considered that compromise of the tumor's

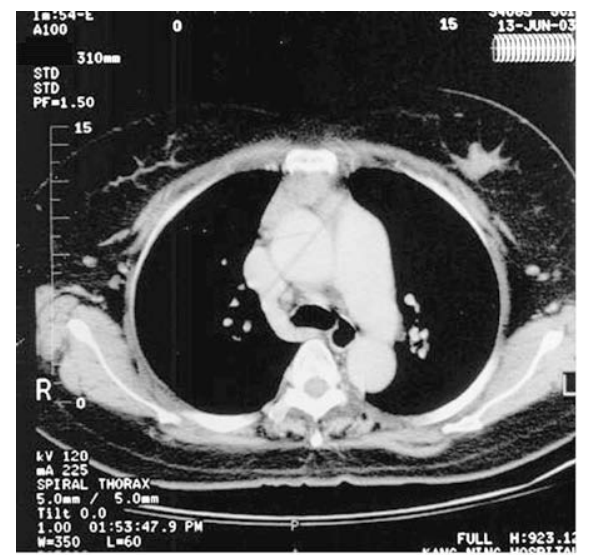

A

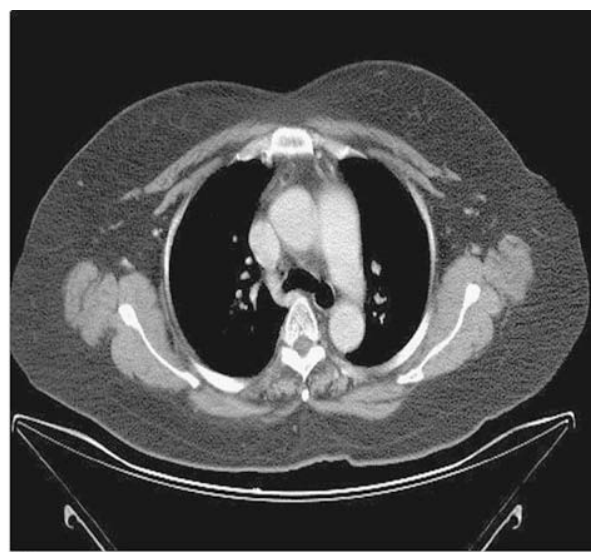

B

FIGURE 1. A, Soft tissue mass about $2.5 \times 2 \mathrm{~cm}$ is seen in prevascular space on computed tomography. B, No definite mediastinal tumor is demonstrated on computed tomography. 


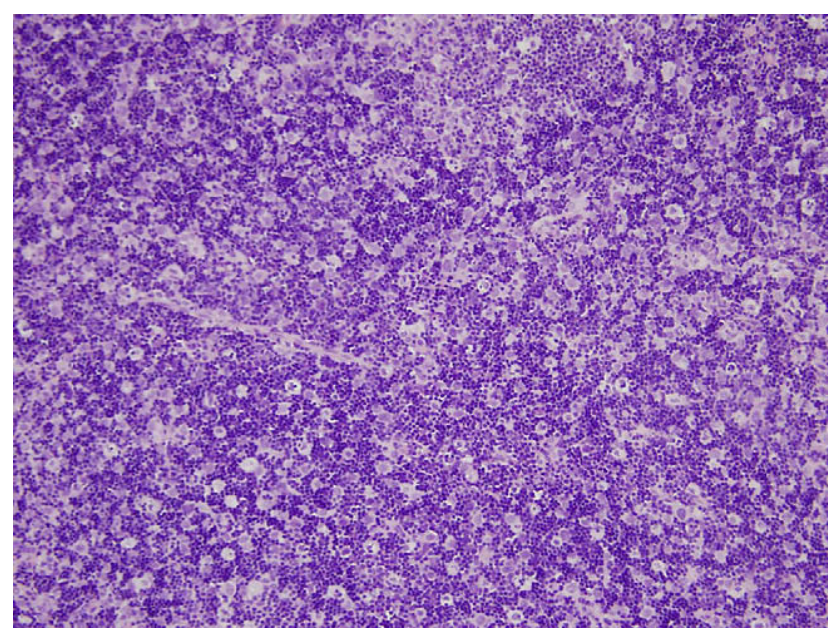

FIGURE 2. Tumor is characterized by large, coarse lobules of tumor with delicate septa, resembling lobular architecture of thymus cortex. Neoplastic tumor cells with polygonal nuclei are intermixed with lymphocytes (hematoxylin and eosin stain; original magnification $\times 200$ ). Histologic analysis was consistent with type B2 thymoma.

blood supply as a result of its rapid enlargement was among the causes of the necrosis. Ten cases of spontaneous regression of thymoma have been reported in the Japaneselanguage literature. ${ }^{3}$ In all cases, the bulk of each tumor was necrotic, with regression being thought to be related to the necrosis. Most thymomas with spontaneous regression are encapsulated, and their prognoses are good.

Apparent spontaneous regression of various malignant neoplasms has been described, although as an unusual phenomenon. Most of these patients had renal cell carcinoma, malignant melanoma, or neuroblastoma. Immunologic factors have been described as playing a significant role. ${ }^{4}$ The spontaneous regression of thymoma has rarely been reported.

We report here the case of a patient with an anterior mediastinal thymoma. The mediastinal thymoma regressed spontaneously 3 years after diagnosis without treatment. We did not obtain specimens to determine the pathologic findings after tumor regression. In this case, the spontaneous regression was seen as the complete disappearance of the tumor on radiologic imaging, with no observed intervening decrease in tumor size. This is different from previous reports. Initially, the patient did not have any symptoms or pleural effusion on follow-up chest radiography. The phenomenon of spontaneous regression may not be related to rapid enlargement and necrosis, and the actual mechanism of spontaneous regression of this thymoma is not clear. This may be clarified in a collection of similar cases. The regression of an invasive thymoma has been reported after corticosteroid therapy. ${ }^{5}$ Our patient did not receive any medical treatment, however, including steroids. Complete spontaneous regression of mediastinal thymomas is possible. Although the phenomenon is rare, conservative treatment may be an alternative to more aggressive therapy for patients with thymoma who are medically unfit.

\section{References}

1. Kuo T. Sclerosing thymoma-a possible phenomenon of regression. Histopathology. 1994;25:289-91.

2. Okagawa T, Uchida T, Suyama M. Thymoma with spontaneous regression and disappearance of pleural effusion. Gen Thorac Cardiovasc Surg. 2007;55:515-7.

3. Ishibashi H, Ashino Y, Niikawa H, Hosaka T, Hasumi T, Shibuya J, et al. [Spontaneous regression of thymoma; report of a case.] [Japanese]. Kyobu Geka. 2003;56:801-5.

4. Cole WH. Spontaneous regression of cancer: the metabolic triumph of the host? Ann N Y Acad Sci. 1974;230:111-41

5. Barratt S, Puthucheary ZA, Plummeridge M. Complete regression of a thymoma to glucocorticoids, commenced for palliation of symptoms. Eur J Cardiothorac Surg. 2007;31:1142-3.

\title{
Acute myocardial infarction and limb ischemia as manifestation of nonbacterial thrombotic endocarditis
}

\author{
Montreal, Quebec, Canada \\ We report a 29-year-old patient who underwent right \\ femoropopliteal bypass 2 years previously for right leg \\ From the Divisions of Cardiac Surgery ${ }^{\mathrm{a}}$ and Cardiology, ${ }^{\mathrm{b}}$ Jewish General Hospital, \\ Montreal, Quebec, Canada. \\ Received for publication March 17, 2008; accepted for publication April 21, 2008. \\ Address for reprints: Jean-François Morin, MD, FRCS, Division of Cardiac Surgery, \\ Jewish General Hospital, A520, 3755 Chemin Côte-Ste-Catherine, Montreal, Que- \\ bec, Canada H3T 1E2 (E-mail: jmorin@surg.jgh.mcgill.ca). \\ J Thorac Cardiovasc Surg 2009; 137:1278-80 \\ $0022-5223 / \$ 36.00$ \\ Copyright (c) 2009 by The American Association for Thoracic Surgery \\ doi:10.1016/j.jtcvs.2008.04.013
}

Naoko Nagano, MD, ${ }^{\mathrm{a}}$ Jean-François Morin, MD, FRCS, ${ }^{\mathrm{a}}$ Dominique Joyal, MD, ${ }^{\mathrm{b}}$ and Lawrence Rudski, MD,

ischemia and was admitted recently to the hospital for acute myocardial infarction secondary to nonbacterial thrombotic endocarditis.

\section{CLINICAL SUMMARY}

A 29-year-old man was admitted to an emergency department with acute onset chest pain. His electrocardiogram showed typical anterior acute myocardial infarction with remarkable ST segment elevations. Emergency coronary angiography was performed, which revealed a complete 